\title{
Qualitative Analysis of Peripheral Peri- Implant Bone and Influence of Alendronate Sodium on Early Bone Regeneration
}

Stephen J. Meraw* and Charles M. Reeve ${ }^{\dagger}$

Background: Alendronate sodium increases alveolar bone density with systemic use. It inhibits osteoclast activity and is thought to result in a net increase in osteoblastic activity. However, little is known about local in vivo use. The purpose of this study was to evaluate the effect of local delivery of alendronate on bone regeneration within peri-implant defects. Peri-implant bone was examined histomorphometrically to evaluate the amount of supporting bone peripheral to the bone-implant interface.

Methods: Six adult hound dogs were evenly divided into 2 groups, with one group receiving alendronate-coated dental implants and the other group serving as controls. Dental implants were placed immediately after extraction of right and left second, third, and fourth mandibular premolars. Forty-eight dental implants were placed ( 2 types in each dog: 24 hydroxyapatite [HA]-coated and 24 titanium machine-polished [TMP]), for a total of 4 variables. A bioabsorbable collagen membrane was secured over the implants and defects, and the flaps closed primarily. The dogs were sacrificed on day 28. Specimens were sectioned, mounted, and stained with Stevenel's blue and van Gieson's picric fuchsin. The amount of bone adjacent and $1 \mathrm{~mm}$ peripheral to the implant surface was recorded with a computerized microscopic digitizer.

Results: Locally applied alendronate resulted in significantly increased amounts of bone $(P<0.0002$, ANOVA) in the peripheral area with both HA and TMP implants. However, the most influential factor in the amount of peripheral bone was the type of implant surface $(P<0.0001)$.

Conclusions: Local application of alendronate is useful in increasing the amount of peripheral peri-implant bone. Also, the amount of supporting bone was not related to the bone-to-implant contact but to the surface characteristics of the implant. The findings of the present study indicate that the evaluation of dental implant-supporting bone should include peripheral bone as well as bone-to-implant interface. J Periodontol 1999;70:1228-1233.

\section{KEYWORDS}

Peri-implantitis/drug therapy; bone regeneration; alveolar bone; alendronate/therapeutic use; animal studies.

\footnotetext{
* Previously, The Mayo Clinic Section of Periodontics, Department of Dental Specialties, Mayo Clinic and Mayo Foundation, Rochester, MN; currently, Department of Periodontics, Prevention and Geriatrics, University of Michigan, Ann Arbor, MI.

$\uparrow$ The Mayo Clinic Section of Periodontics.
}

uccessful long-term use of
dental implants depends on
integration within, and support from, osseous tissues. Various morphologic features of alveolar bone have been suggested to exist within individuals and to be associated with various regions of the oral cavity. ${ }^{1,2}$ These alveolar bone "types" have also been associated with varying long-term success of dental implants. ${ }^{3}$ One example is the Lekholm and Zarb classification of type IV bone, which is composed of widely spread trabeculae with large marrow spaces and little cortical support. ${ }^{1}$ This type of bone has been associated with lower rates of implant success because of inadequate bony support. ${ }^{3}$

Evaluation of implant surgery success before prosthetic loading includes determination of osseointegration. Clinically, this usually is associated with lack of mobility of the implant fixture after adequate healing. Histologically, osseointegration has been described as direct attachment or connection of vital osseous tissues to the surface of the implant, without intervening connective tissue. ${ }^{4}$ Histologic evaluation of osseointegration usually involves a description of the percentage of bone-to-implant contact; however, this does not fully describe the quality of the bone support. 
Modification and regeneration of osseous tissues have been an ongoing area of research. The improvement of bone regeneration has included the use of biologic mediators to improve the quantity and quality of the bone being regenerated. ${ }^{5-10}$ One group of bone metabolism mediators is the bisphosphonates, which are believed to inhibit osteoclast activity by interfering with the ruffled border membrane of the osteoclasts, without destroying the cells. ${ }^{11}$ This inhibition of resorptive activity is thought to produce a shift in bone turnover equilibrium to more osteoblastic activity. Previous studies have demonstrated a positive effect with the bisphosphonate alendronate sodium,$^{\ddagger}$ and the systemic use of this agent in animal models has resulted in decreased bone loss and increased bone density of alveolar bone. ${ }^{12-16}$

The use of biologic bone mediators in the improvement of poor quality bone potentially could help improve the success of dental implants. The aim of this study was to evaluate effects of alendronate within areas of bone regeneration around dental implants. Because severe side effects have been reported with systemic use of alendronate in humans, ${ }^{17}$ local delivery methods were used to avoid or to reduce the possibility of adverse systemic effects. Furthermore, histologic evaluation of the peri-implant bone included a qualitative description of the supportive bone beyond the bone-to-implant interface.

\section{MATERIALS AND METHODS}

Six adult male hound dogs were used in this study because jaw size was adequate for endosteal implant placement. This study was approved by the Institutional Animal Care and Use Committee of the Mayo Foundation. Forty-eight implants were used and distributed evenly among 4 treatment groups: hydroxyapatite (HA)-coated implants $\S$ with alendronate, titanium machine-polished (TMP) implants" with alendronate, HA-coated implants without alendronate, and TMP implants without alendronate. There were 4 implants per mandible side, with 12 implants per treatment variable. Implant treatment groups were allocated per mandible side and not staggered per position on a given side, because of the proximity of implants on the same side and the possibility of cross-reactivity with proximity.

HA-coated implants for the alendronate group were loaded with alendronate similar to the technique described by Denissen et al., ${ }^{18}$ in which HA-coated implants were soaked in a $0.1 \mathrm{mmol}$ sterile, neutral solution of alendronate for 1 week at $37^{\circ} \mathrm{C}$, which was shown to have a slow, constant release of other bisphosphonates from HA over time. This concentration has been shown to be bioactive and not to have cytotoxic effects in vitro. ${ }^{19}$
TMP implants were coated 1 with $2.8 \mu \mathrm{g}$ of alendronate, which is approximately the amount released over a 1 -month period. ${ }^{18}$ This second model was developed for application in implants without an HA coating as well as for comparing surface coating to a known time-release model. TMP implants coated with alendronate were resterilized with the ethylene oxide method before surgical placement.

At the time of surgery, each dog was given $4 \%$ thiamylan sodium, $20 \mathrm{mg} / \mathrm{kg}$ intravenously. ${ }^{\#}$ After induction of general anesthesia, the animal was intubated and maintained with $1 \%$ halothane inhalational anesthetic in conjunction with a $50 \%$ mixture of nitrous oxide and oxygen. The surgical sites were disinfected with a sterile swab of povidone-iodine** and anesthetized locally with $2 \%$ lidocaine $\mathrm{HCl}$ with $1: 100,000$ epinephrine for improved hemostasis and postoperative analgesia. Full-thickness mucoperiosteal flaps were incised and reflected on the facial and lingual aspects of the teeth to be extracted. The second, third, and fourth mandibular premolar teeth were extracted bilaterally as atraumatically as possible with midcoronal facial-tolingual sectioning with a high-speed handpiece with a sterile water-cooled bur and standard dental forceps. After odontectomy, alveoloplasty was performed on the remaining alveolar ridge with use of a bone file for elimination of bony spicules and improved form before implant placement. No dehiscences were present. Next, 4 endosteal dental implants were surgically placed in each side of the mandible with sterile water cooling, according to assigned modality. Cover screws were placed to cover the implants. Defects remained greater than $1 \mathrm{~mm}$ from the implant surface in the coronal half of the implants. A biabsorbable collagen membrane ${ }^{\dagger \dagger}$ was placed over the sites to prevent epithelial downgrowth. ${ }^{20}$ The membranes were secured in place with bone tacks ${ }^{\ddagger \ddagger}$ placed at each corner of the membrane to prevent movement of the membrane. No bone replacement graft was used. Surgical flaps was reapproximated and closed with 4-0 polyglactin 910 suture. $\$ \S$

Postoperatively, butorphanol,,\|\|$\| .2$ to $0.4 \mathrm{mg} / \mathrm{kg}$ every 2 to 5 hours, was administered intramuscularly as needed for discomfort, and a 300,000 IU/ml preparation of penicillin $G$ benzathine and penicillin $G$ procaine, , 1 l $1 \mathrm{ml} / 5$ to $10 \mathrm{~kg}$, was given intramuscularly to reduce potential of infection. The dogs were kept in the recovery room until they overcame the

‡ Fosamax, Merck \& Co., Inc., West Point, PA

$\S$ ThreadLoc, Sulzer Calcitek, Carlsbad, CA.

I| Brånemark, Nobel BioCare, Westmont, IL.

I Surface Genesis, Sunnyvale, CA.

\# Surital, Parke-Davis Co., Detroit, MI.

** Betadine, Purdue Frederick Co., Newport, CT.

$\dagger \dagger$ Biomend, Calcitek, Carlsbad, CA.

† IMTEC, Ardmore, OK.

$\S \S$ Vicryl, Ethicon, Inc., Johnson \& Johnson Co., Sommerville, NJ.

III Torbugesic, Aveco Co. Inc., Fort Dodge, IA.

II Flo-Cillin, Fort Dodge Laboratories, Inc., Fort Dodge, IA. 
effects of general anesthesia and their condition was stable. For the rest of the study, they were fed a soft diet $^{\# \#}$ to reduce potential trauma to the surgical sites.

Histologic examination occurred after a 1month healing period. This period was chosen to evaluate any early differences when the maximal amount of alendronate was likely present. Statistical comparisons were made between the use of HA-coated and TMP implants with alendronate and HAcoated and TMP implants without alendronate.

The specimens were sectioned, fixed in $70 \%$ ethanol for 1 day, and then dehydrated in successive concentrations of alcohol and GMA (2-hydroxyethylmethacrylate). Plastic infiltration of the specimens was accomplished with an even mixture of GMA and embedding medium, ${ }^{* * *}$ followed by repeated immersions in $100 \%$ embedding medium. A microgrinding system ${ }^{\dagger \dagger \dagger}$ was used to obtain sections less than $50 \mu \mathrm{m}$ thick. ${ }^{21}$

Histologic specimens were examined with transmitted light at a magnification of $\times 100$, and the implant and peri-implant tissues, including amount of bone per area, were analyzed with the use of a semi-automated computerized technique. ${ }^{\neq} \neq$The amount of bone per area in each field $\left(0.507 \mathrm{~mm}^{2}\right)$ was traced with the digitizer and recorded as an area value, 2 fields from the implant surface, along the length of the implant. This value was then converted to a mean percentage of the total area per field.

\section{Statistical Analysis}

The primary response variable was percentage of bone per area along the length of the implant section, as measured histologically. Multifactor analysis of variance (ANOVA) was used to identify effects due to alendronate, HA coating, and their possible interaction. Effects were considered significant at alpha $=$ 0.05 .

\section{RESULTS}

The results of ANOVA for the histomorphometric data are summarized in Table 1 . The primary response variable was percentage of bone per area, as measured histologically (Fig. 1).

In general, all 48 implants were clinically integrated at the time of retrieval; none was mobile, infected, or lost. No adverse systemic effects were encountered in terms of intraoral mucosal ulcerations or gastrointestinal distress.

For percentage of bone per area, alendronate had a significant effect $(P>0.0002)$, with a mean increase in bone per area of $5.8 \%$ (Fig. 2). However, greater differences existed between type of implant surface and percentage of bone per area and were significant $(P$
$>0.0001)$. TMP implants demonstrated a $9.5 \%$ mean increase in bone per area compared with HA-coated implants (Fig. 3).

Of the 4 treatment combinations, alendronate-TMP implants demonstrated the greatest mean bone per area, and control-HA implants showed the least.

\section{DISCUSSION}

The present study examined the effects of locally applied alendronate on early bone regeneration in peri-implant defects. Evaluation of the effects involved histometric analysis of the peripheral implant-supporting bone, up to $1 \mathrm{~mm}$ from the threaded surface. Evaluation of systemic effects was limited to clinical observations, through which no adverse side effect was noted in terms of intraoral mucosal ulcerations or gastrointestinal distress.

The mean percentage of supporting bone per area was improved in both of the local alendronate models. The mean improvement of $5.8 \%$ is slightly less than that in previous reports, which evaluated changes of bone density radiographically with systemic use of alendronate. ${ }^{12,22}$ Improvements of $7 \%$ to $10 \%$ bone density have been reported for vertebral bone ${ }^{22}$ and $11.6 \%$ greater bone density for alveolar bone with systemic alendronate use. ${ }^{12}$ Direct comparisons may be difficult because the present study used a histometric evaluation instead of a radiographic evaluation, as in previous studies. Also, little is known about local use of alendronate with alveolar bone. Therefore, further testing may be helpful to determine a more optimal local dosage that could lead to improved gains.

The implant surface influenced the amount of bone per area. TMP implants demonstrated a greater mean bone per area than HA implants. The underlying mechanism was not determined in our study. Malecki et al. ${ }^{23}$ reported $8 \%$ greater bone per area with TMP implants than HA implants, although this did not reach statistical significance. This trend is contrary to that of studies which evaluated only the percentage of bone-toimplant contact, in which a superior bone-to-implant

\#\# Science Diet, Hill's Pet Nutrition, Inc., Topeka, KS.

*** Technovit 7200 VLC, Kulzer E Co., Norderstedt, Germany.

$\dagger \dagger \dagger$ EXAKT Medical Instruments, Oklahoma City, OK.

㸬BQ System IV Program, R \& M Biometrics, Inc., Nashville, TN. 

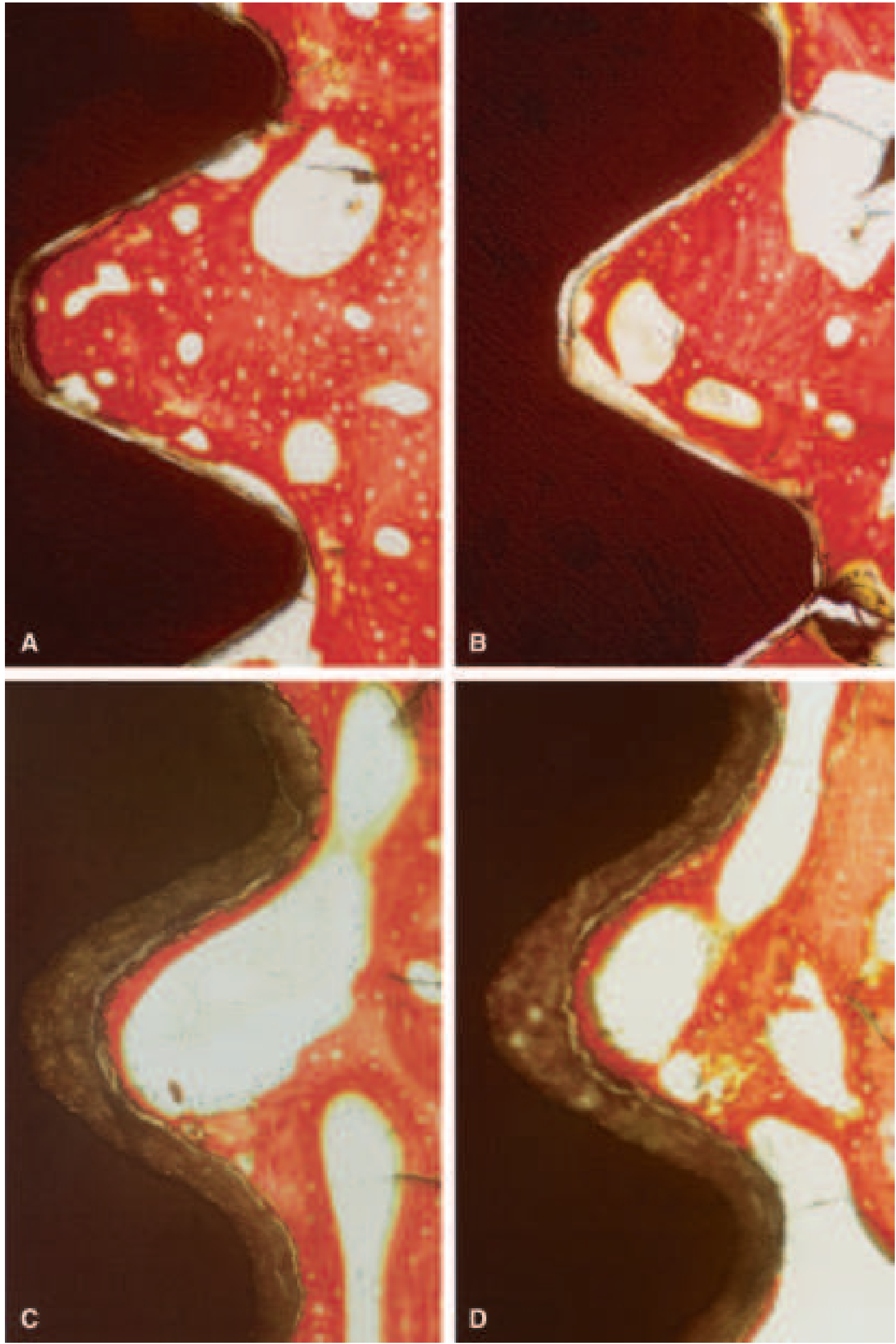

Figure I.

Peripheral bone per area. A) Titanium machine-polished alendronate implant. B) Titanium machine-polished control implant. C) Hydroxyapatite-alendronate implant. D) Hydroxyapatite-control implant (Stevenel's blue and van Gieson's picric fuchsin stain; original magnification x100). 
70

60

50

40

30

20

10

0

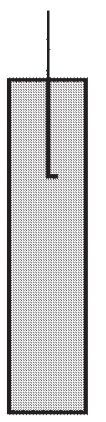

Alendronate $(\mathrm{SD}=8.7)$
Control $(\mathrm{SD}=6.6)$

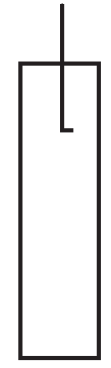

Figure 2.

Percentage of bone per area. Effect of alendronate.

80

70

60

50

40

30

20

10

0

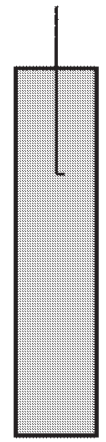

$\mathrm{TMP}(\mathrm{SD}=6.6)$

$\mathrm{HA}(\mathrm{SD}=7.1)$

Figure 3.

Percentage of bone per area. Effect of implant surface.

contact was achieved with HA implants compared to TMP implants in early healing. ${ }^{24-26}$ We previously found a $49.7 \%$ mean bone-to-implant contact for HA implants compared to $39.3 \%$ for TMP implants in an early healing model. ${ }^{24}$ Therefore, reporting only the bone-to-implant contact might not be a complete description in terms of peri-implant supporting bone. Histologic descriptions of peri-implant supporting bone should include both the bone-to-implant contact as well as the peripheral bone per area. This provides a more complete description of the support that the implant receives. Possibly, a high bone-to-implant contact with a low amount of peripheral bone per area might be less favorable than the converse for long-term support and resistance to breakdown. However, additional studies are needed to explore these relationships.

\section{ACKNOWLEDGMENTS}

We thank the Hard Tissue Laboratory in Oklahoma City, especially Drs. Michael Rohrer, Tom Sprowl, and
Hari Prasad for technical assistance; Surface Genesis in Sunnyvale, California for coating the TMP implants; Nobel BioCare and Sulzer-Calcitek for material support; and Ms. Christine M. Lohse for help with the statistical analysis. We also thank the Mayo Foundation and Merck \& Co., Inc., for financial support.

\section{REFERENCES}

1. Lekholm U, Zarb GA. Patient selection and preparation. In: Brånemark PI, Zarb GA, Albrektsson TA, eds. TissueIntegrated Prostheses, Osseointegration in Clinical Dentistry. Chicago: Quintessence Publishing;1985:12.

2. Misch CE. Density of bone: Effect on treatment plans, surgical approach, healing, and progressive bone loading. Int J Oral Implantol 1990;6:23-31.

3. Jaffin RA, Berman CL. The excessive loss of Brånemark fixtures in type IV bone: A 5-year analysis. J Periodontol 1991;62:2-4.

4. Brånemark PI, Hansson $\mathrm{B}$, Adell R, et al. Osseointegrated oral implants in the rehabilitation of the edentulous patient. Experience from a 10-year period. Scand J Plast Reconstr Surg 1977;11(Suppl. 16):1-132.

5. Hauschka PV. Growth factor effects in bone. In: Hall BK, ed. Bone: A Treatise. Caldwell, NJ: Telford Press; 1990; 130-170.

6. Bonewald LF, Mundy GR. Role of transforming growth factor-beta in bone remodeling. Clin Orthop 1990;250: 261-276.

7. Mohan S, Baylink DJ. Bone growth factors. Clin Orthop 1991;263:30-48.

8. Baylink DJ, Finkelman RD, Mohan S. Growth factors to stimulate bone formation. J Bone Miner Res 1993; 8(Suppl. 2):S565-S572.

9. Wozney JM. The bone morphogenetic protein family and osteogenesis. Mol Reprod Dev 1992;32:160-167.

10. The American Academy of Periodontology. The potential role of growth and differentiation factors in periodontal regeneration (Position Paper). J Periodontol 1996;67:545-553.

11. Sato M, Grasser W, Endo N, et al. Bisphosphonate action. Alendronate localization in rat bone and effects on osteoclast ultrastructure. J Clin Invest 1991;88: 2095-2105.

12. Reddy MS, Weatherford TW, Smith CA, West BD, Jeffcoat MK, Jacks TM. Alendronate treatment of naturally-occurring periodontitis in beagle dogs. J Periodontol 1995;66:211-217.

13. Weinreb M, Quartuccio H, Seedor JG, et al. Histomorphometrical analysis of the effects of the bisphosphonate alendronate on bone loss caused by experimental periodontitis in monkeys. J Periodont Res 1994; 29:35-40.

14. Brunsvold MA, Chaves ES, Kornman KS, Aufdemorte TB, Wood R. Effects of bisphosphonate on experimental periodontitis in monkeys. J Periodontol 1992; 63:825-830.

15. Yaffe A, Iztkovich M, Earon Y, Alt I, Lilov R, Binderman I. Local delivery of an amino bisphosphonate prevents the resorptive phase of alveolar bone following mucoperiosteal flap surgery in rats. J Periodontol 1997; 68:884-889.

16. Yaffe A, Fine N, Alt I, Binderman I. Effect of bisphosphonate on alveolar bone resorption following mucoperiosteal flap surgery in the mandible of rats. $J$ Periodontol 1995;66:999-1003. 
17. De Groen PC, Lubbe DF, Hirsch LJ, et al. Esophagitis associated with the use of alendronate. N Engl $J$ Med 1996;335:1016-1021.

18. Denissen H, van Beek E, Lowik C, Papapoulos S, van den Hooff A. Ceramic hydoxyapatite implants for the release of bisphosphonate. Bone Min 1994;25:123-134.

19. Tsuchimoto M, Azuma Y, Higuchi O, et al. Alendronate modulates osteogenesis of human osteoblastic cells in vitro. Jpn J Pharmacol 1994;66:25-33.

20. Hurley LA, Stinchfield FE, Bassett AL, Lyon WH. The role of soft tissues in osteogenesis: An experimental study of canine spine fusions. J Bone Joint Surg 1959; 41:1243-1254.

21. Rohrer MD, Schubert CC. The cutting-grinding technique for histologic preparation of undecalcified bone and bone-anchored implants. Oral Surg Oral Med Oral Pathol 1992;74:73-78.

22. Liberman UA, Weiss SR, Broll J, et al. Effects of oral alendronate on bone mineral density and the incidence of fractures in postmenopausal osteoporosis. N Engl J Med 1995;333:1437-1443.

23. Malecki CM, Mitchell JC, Murdock GP, Carr AB. Histomorphometric analysis of three implant materials using an automated image analysis system. J Dent Res 1998;77(Spec. Issue):172(Abstr. 534).
24. Meraw SJ, Reeve CM, Wollan PC. Use of alendronate in peri-implant defect regeneration. J Periodontol 1999; 70:151-158.

25. Carlsson L, Regner L, Johansson C, Gottlander M, Herberts P. Bone response to hydroxyapatite-coated and commercially pure titanium implants in the human arthritic knee. J Orthop Res 1994;12:274-285.

26. Gottlander M, Albrektsson T. Histomorphometric studies of hydroxylapatite-coated and uncoated CP titanium threaded implants in bone. Int $J$ Oral Maxillofac Implants 1991;6:399-404.

Send reprint requests to: Dr. Stephen Meraw, Department of Periodontics, Prevention and Geriatrics, School of Dentistry, University of Michigan, 1011 N. University Ave., Ann Arbor, MI 48109-1078.

Accepted for publication February 16, 1999. 